\title{
DRG2 Regulates G2/M Progression via the Cyclin B1-Cdk1 Complex
}

\author{
Soo Hwa Jang ${ }^{1}$, Ah-Ram Kim ${ }^{1}$, Neung-Hwa Park ${ }^{2}$, Jeong Woo Park ${ }^{1, *}$, and In-Seob Han ${ }^{1, *}$
}

\begin{abstract}
Developmentally regulated GTP-binding protein 2 (DRG2) plays an important role in cell growth. Here we explored the linkage between DRG2 and G2/M phase checkpoint function in cell cycle progression. We observed that knockdown of DRG2 in HeLa cells affected growth in a wound-healing assay, and tumorigenicity in nude mice xenografts. Flow cytometry assays and $\left.{ }^{3} \mathrm{H}\right]$ incorporation assays indicated that $\mathrm{G} 2 / \mathrm{M}$ phase arrest was responsible for the decreased proliferation of these cells. Knockdown of DRG2 elicited down-regulation of the major mitotic promoting factor, the cyclin B1/Cdk1 complex, but upregulation of the cell cycle arresting proteins, Wee1, Myt1, and p21. These findings identify a novel role of DRG2 in G2/M progression.
\end{abstract}

\section{INTRODUCTION}

Developmentally regulated GTP-binding protein 2 (DRG2) is a member of a subfamily of the GTPase superfamily that is involved in the control of cell growth and differentiation. DRG2 plays a critical role in control of the cell cycle and apoptosis in Jurkat T cells (Ko et al., 2004). Although we have previously demonstrated the importance of DRG2 in cell cycle arrest in the G2/M phase, it remains unclear just how it regulates mitosis (Song et al., 2004).

Progression into mitosis is an accurately regulated process ultimately driven by the cyclin B1/cyclin dependent kinase 1 (Cdk1) (Fisher et al., 2012). In normal cells, the cyclin B1/Cdk1 complex remains inactive due to inhibitory phosphorylation of Cdk1 until the end of $\mathrm{G}_{2}$ phase. Dephosphorylation of $\mathrm{Cdk} 1$ by one of the Cdc25 family of phosphatases initiates entry into mitosis. In contrast, Wee1 inactivates the cyclin B1/Cdk1 complex by phosphorylation (Fisher et al., 2012). A substantial amount of cyclin B1/Cdk1 also remains in the cytoplasm during mitosis, and active cyclinB1/Cdk1 kinase is found both in the cytoplasm and nucleus during prophase (Gavet and Pines,

\footnotetext{
${ }^{1}$ School of Biological Sciences, University of Ulsan, Ulsan 44610, Korea, ${ }^{2}$ Department of Internal Medicine, University of Ulsan College of Medicine, Ulsan University Hospital, Ulsan 44033, Korea

*Correspondence: hanis@ulsan.ac.kr (ISH); jwpark@ulsan.ac.kr (JWP)
}

Received 14 June, 2016; revised 25 August, 2016; accepted 25 August, 2016; published online 27 September, 2016

Keywords: cyclin B/Cdk1 complex, DRG2, G2/M check point, p21
2010). Cdk1 is activated by binding one of the cyclin family members that are sequentially expressed during the cell cycle. Cdk1 is inhibited when bound to Cdk inhibitory proteins (CKIs), including members of the $\mathrm{p} 21$ family.

It is well known that the cyclin-dependent kinase inhibitor p21 protein plays an important role in arrest in the $\mathrm{G} 2$ phase of the cell cycle (Cazzalini et al., 2010). Regulation of p21 relies basically on two posttranslational modifications, namely phosphorylation and ubiquitination. The main role of p21 in cell-cycle regulation is inhibiting the activity of cyclin B/Cdk1 complexes. p21 is also thought to be responsible for nuclear retention of the cyclin B/Cdk1 complex and cyclin B degradation in response to DNA damage (Charrier-Savournin et al., 2004). In addition to its function as a Cdk inhibitor, p21 has been recently shown to contribute to cell cycle arrest through transcriptional repression of cell cycle regulatory genes (Cazzalini et al., 2010). Thus, p21 controls the checkpoint preventing G2-arrested cells from mitotic entry through multiple mechanisms.

In this study, we examined the biochemical properties of DRG2 with respect to cell cycle-associated activity. We used DRG2 knockdown HeLa cells to determine how DRG2 is involved in arrest of the cell cycle in G2/M.

\section{MATERIALS AND METHODS}

Cell culture and cell counting

Stable DRG2 knockdown HeLa cells were previously established by transfection of shRNA (Mani et al., 2016). Briefly, the MISSION ${ }^{\circledR}$ pLKO-empty vector and a vector encoding pLKOshRNA against DRG2 (Sigma, USA) were transfected into HeLa cells with lipofectamine 2000 (Life Technology, USA). Cells were maintained in DMEM supplemented with $10 \%$ fetal bovine serum, $100 \mathrm{U} / \mathrm{ml}$ penicillin-streptomycin solution and 1 $\mu \mathrm{g} / \mathrm{ml}$ puromycin (Sigma) to select against non-transfected cells. The medium was changed every other day until resistant colonies could be identified. After the antibiotic selection, cells were maintained under standard conditions without puromycin in a humidified atmosphere of $95 \%$ air $/ 5 \% \mathrm{CO}_{2}$ at $37^{\circ} \mathrm{C}$. The efficiency of DRG2 knockdown was validated by RT-PCR and Western blot analysis (Fig. 1A).

\section{Cell synchronization and cell cycle analysis}

To synchronize cells at the G1/S boundary, cells were seeded in $100 \mathrm{~mm}$ cell culture dishes at $2 \times 10^{4} \mathrm{cells} / \mathrm{ml}$. After 1 day, they were transferred to a $2 \mathrm{mM}$ thymidine solution and incubated for $18 \mathrm{~h}$. For release from thymidine block, the cells were incubated with fresh medium for $8 \mathrm{~h}$ and blocked again with 
A

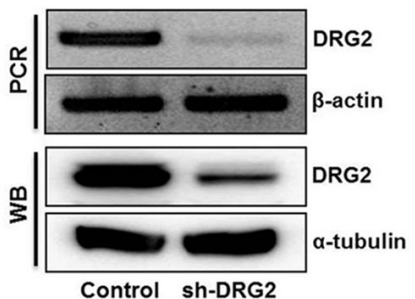

Control sh-DRG2
B
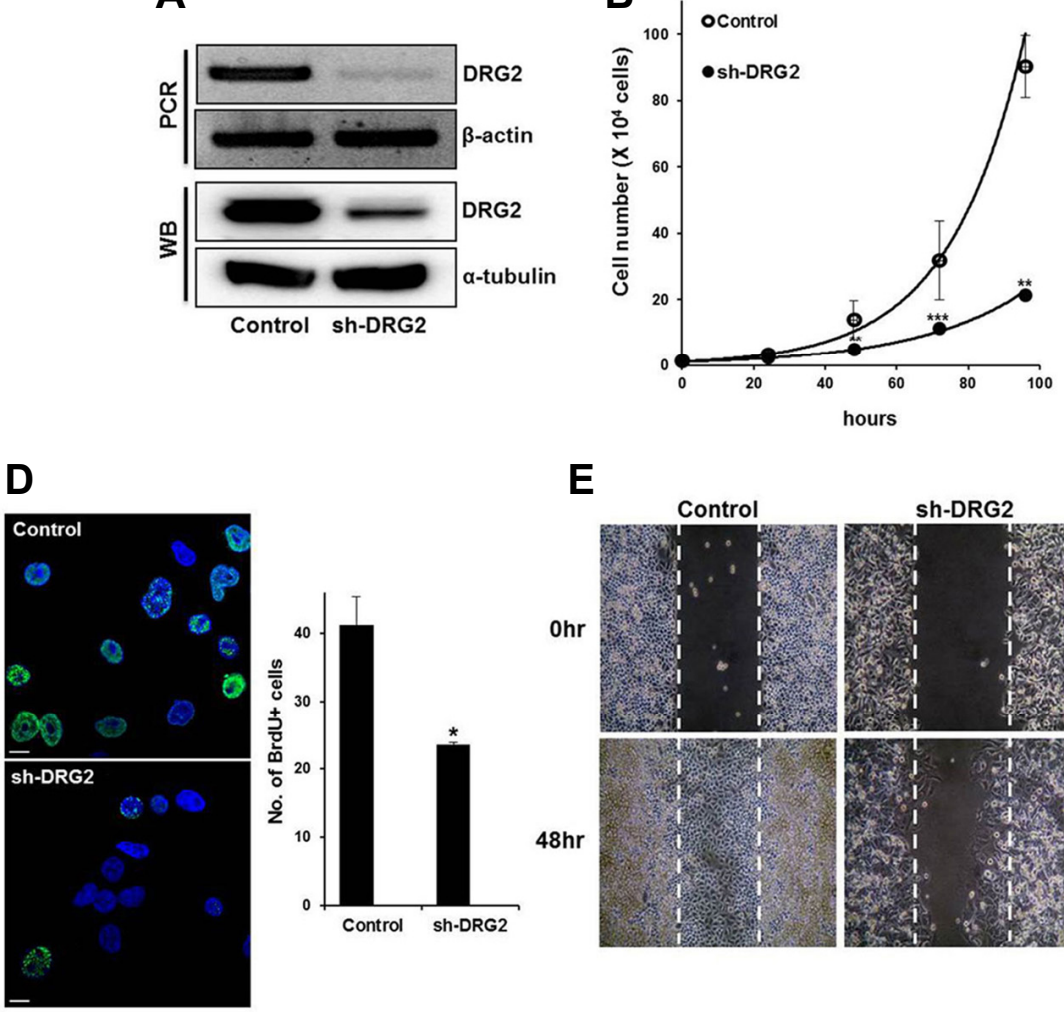

E

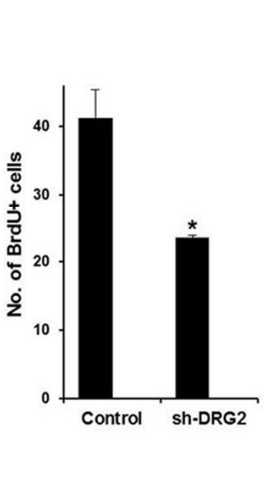

C

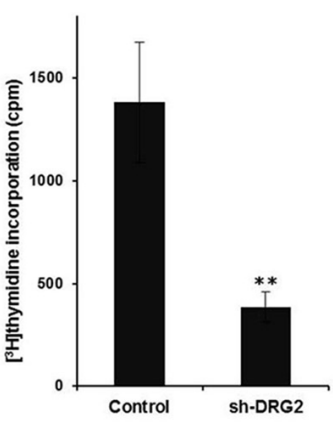

$\mathbf{F}$
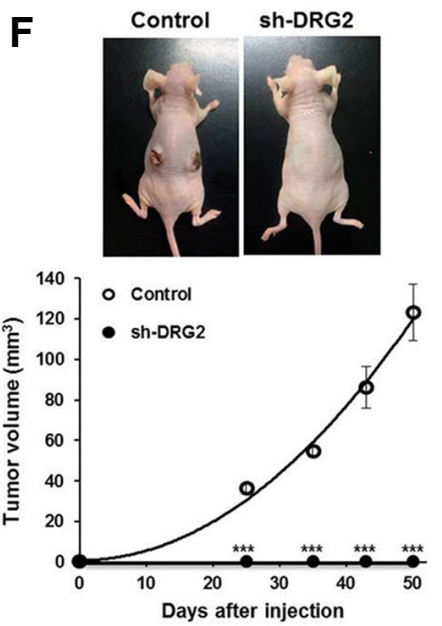

Fig. 1. Depletion of DRG2 inhibits HeLa cell proliferation. (A) Knock-down efficiency of the sh-DRG2 cells. (B) Cell proliferation was determined by cell counting. Data are means \pm S.E. of three independent experiments $\left({ }^{* *} p<0.01 ;{ }^{* * *} p<0.001\right)$. (C) Cells were incubated with $\left[{ }^{3} \mathrm{H}\right]$-thymidine for 20 hours and the incorporated tritium was measured with a beta counter. Data are means \pm S.E. of three independent experiments. (D) Number of BrdU positive cells was reduced in sh-DRG2 cells. Data are presented as the mean \pm S.E. $\left({ }^{*} p<0.05\right)(G r e e n ; B r d U$, Blue; DAPI, Scale bar; $10 \mu \mathrm{m}$ ). (E) Cell migration was assessed by a wound healing assay using silicon inserts. The dashed line represents time zero, and the denuded area is gradually closed by cell migration. $(F)$ Suppression of in vivo tumor development by DRG2 deficiency. Control and sh-DRG2 cells were injected subcutaneously into BALB/c nude mice ( $n=4$ for each group). Data present mean $\pm S$.E. of two independent experiments. Photographs of representative mice were taken 8 weeks after injection. See "Materials and Methods" for further details.

$2 \mathrm{mM}$ thymidine for $16 \mathrm{~h}$. After that they were released from thymidine block, collected at the indicated time points and fixed in ice-cold $70 \%$ ethanol for $12 \mathrm{~h}$ at $-20^{\circ} \mathrm{C}$. The fixed cells were washed with PBS and incubated with $50 \mu \mathrm{g} / \mathrm{ml}$ RNase A solution (Amresco, USA) to remove RNA. To measure DNA contents, $40 \mu \mathrm{g} / \mathrm{ml}$ of propodium iodide (Sigma) was added and DNA was measured with a BD FACSCanto II (BD Bioscience, USA). Data were analyzed with Flowjo software (v. 7.1).

\section{Wound healing assay}

Culture inserts (Ibidi $\mathrm{GmbH}$, Germany) were adapted for identifying the effect of DRG2 on cell migration. Silicon-inserts have two reservoirs that are separated by a $500 \mu \mathrm{m}$ thick wall that creates a wound gap of constant size and generates little mechanical cell stress compared with the typical scratch woundhealing assay. The silicon-inserts were tightly positioned in 12well cell culture plates, and $3 \times 10^{4}$ cells were seeded in both reservoirs. Next day, the inserts were carefully detached and washed gently with medium to prevent the floating cells from re-attaching in the cell-free zone. Cell migration was monitored from the reduction in the $500 \mu \mathrm{m}$-wide cell-free zone at a given time.

\section{$\left[{ }^{3} \mathrm{H}\right]$-thymidine incorporation assay}

Multiple cell samples were plated in the 96-well cell culture plate $\left(8 \times 10^{3}\right.$ cells/well). Next day, $1.25 \mu \mathrm{Ci}$ of $\left[{ }^{3} \mathrm{H}\right]$ thymidine (PerkinElmer, USA) was added to each well and incubation continued for $20 \mathrm{~h}$. To detect the label incorporated into DNA, the cells were harvested onto glass fiber filter membranes (PerkinElmer) using a cell harvester. The membranes were allowed to air-dry for 3-5 $\mathrm{h}$ at room temperature and radioactivity was counted with a beta counter (Wallac, USA).

\section{Western blot analysis}

Cells were lysed in $1 \times$ passive lysis buffer (Promega, USA) and protein was quantified using a BCA protein assay kit (Pierce, USA). Aliquots containing approximately $30 \mu \mathrm{g}$ of protein were resolved on $10-12 \%$ SDS-polyacrylamide gels and 
A
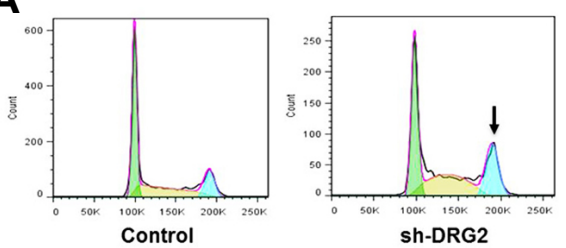

\begin{tabular}{ccc}
\hline & Control & sh-DRG2 \\
G0/G1 & $49.00 \pm 2.38$ & $44.51 \pm 2.87$ \\
$\mathrm{~S}$ & $34.45 \pm 2.99$ & $30.97 \pm 3.30$ \\
$\mathrm{G} 2 / \mathrm{M}$ & $16.54 \pm 0.68$ & $24.51 \pm 1.50^{\text {** }}$ \\
\hline
\end{tabular}

B

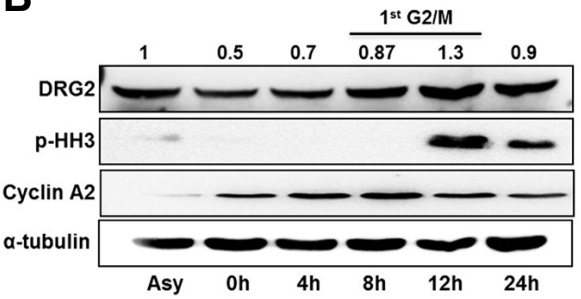

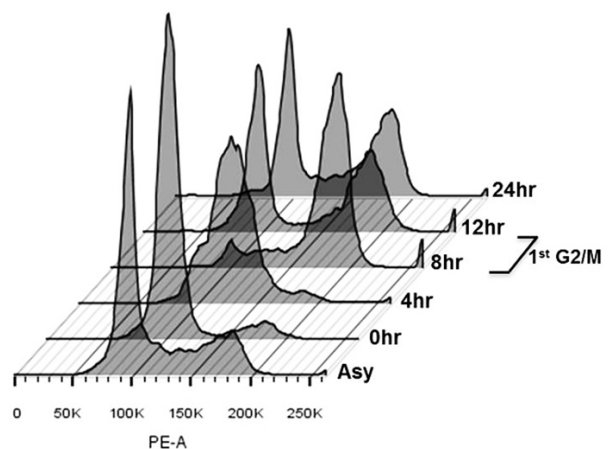

Fig. 2. Depletion of DRG2 perturbs normal cell cycle progression. (A) Exponentially growing cells were harvested and DNA content was measured using propodium iodide and flow cytometry. The G2/M phase population was significantly increased in sh-DRG2 cells. Data are means \pm S.E. of eight independent experiments. (B) Cell cycle-dependent expression of DRG2 was determined by the doublethymidine synchronization method. Cyclin A2 and pHH3 were used as markers of G2/M phase. Data are means of three independent experiments; band intensities were analyzed using Image J program. transferred to PVDF membranes (Pall Corporation, USA). The membranes were blocked with TBS-Tween 20 containing 5\% non-fat milk for $1 \mathrm{~h}$ and probed with antibodies to DRG2 (Sigma), cyclin A2, cyclin B1, Cdk1, phospho-Cdk1(Tyr15), Myt1, phospho-Wee1(Ser642) and phospho-Histone H3 (Ser10) (Cell Signaling Technology $\left.{ }^{\circledR}, \mathrm{USA}\right)$, followed by horseradish peroxidase-conjugated secondary antibody (Santa Cruz Biotechnology, USA). Blots were visualized with SuperSignal West Pico Chemiluminescent Substrate (Thermo Fisher Scientific Inc, USA), and band intensities were measured using Image $\mathrm{J}$ software $1.42 \mathrm{q}(\mathrm{NIH}, \mathrm{USA})$.

\section{Plasmid DNA transfection}

p3XFLAG shRNA-resist hDRG2 construct was kindly provided by Dr. Sung Hoon Back (University of Ulsan) and used to rescue of DRG2 in sh-DRG2 cells. To establish the shRNA resistant DRG2 clone, silent mutation was employed. p3XFLAG (without rescue DNA) vector was used as a negative control. After seeding the cells in the 6-well cell culture plate, vector and shRNAresist-hDRG2 plasmid DNA were transfected using Lipofectamine 3000 according to manufacturer's instruction. Next day, cells were harvested for Western blot analysis.

\section{Immunofluorescence}

Cells were plated onto 8-well Lab-Teck chamber slide (Thermo Fisher Scientific Inc). Next day, cells were washed with PBS and fixed with $4 \%$ paraformaldehyde for $15 \mathrm{~min}$ at roomtemperature. For permeabilization, cells were exposed to $0.1 \%$ Triton $\mathrm{X}-100$ for 10 min and blocked with $5 \%$ normal goat serum. Following incubation with primary antibodies against cyclin B1, Cdk1, pCdk1 (Tyr15), p21 (Cell signaling Technology) and DRG2 (abcam, USA), they were transferred to TBS containing $0.05 \%$ Triton $\mathrm{X}-100$ and $0.1 \%$ Tween 20 overnight at $4^{\circ} \mathrm{C}$. Signals were visualized with Alexa 488- or Alexa 594-conjugated secondary antibodies (Life Technology) and images were acquired using a FV1000 confocal microscope (Olympus, Japan).
Bromodeoxyuridine (BrdU) assay

Cells were plated $4 \times 10^{4}$ cells/well in 4-well Lab-Teck chamber slide (Thermo Fisher Scientific Inc.). To detect newly synthesized DNA, $10 \mu \mathrm{M}$ of BrdU was treated for 3 hours and then fixed with $4 \%$ paraformaldehyde, serum-blocked, and stained with anti-BrdU antibody (Cell signaling). Next day, cells were reacted with alexa 488-conjugated secondary antibodies and images were captured using FV1000 Confocal Microscope (Olympus, Japan).

\section{Xenograft model}

All animal experiments were performed in accordance with procedures approved by the Institute of Laboratory Animal Resources, University of Ulsan. Six-week-old male CAnN.CgFoxn $1^{\text {nu }} /$ CrijOri nude mice were purchased from Orient Bio Inc. (Korea). For adaptation, mice were housed for 1 week and randomly divided into two groups. Both control and sh-DRG2 cells $\left(1 \times 10^{5}\right.$ cells in $10 \%$ DMEN) were injected subcutaneously into the left and right sides, of the flanks of mice anesthetized with $2.5 \%$ 3-bromoethanol into each groups. Tumor volumes and body weights were evaluated every 1 week using the formula: volume $=($ width $\times$ length $\times$ depth $) \times(\pi / 6)$.

\section{RESULTS}

DRG2 knockdown impairs the proliferation of HeLa cells in vitro and in vivo

To characterize the roles of DRG2 in cell proliferation, we generated DRG2 knockdown HeLa cells (named sh-DRG2) and examined their growth in vitro and in vivo. The growth rate of the sh-DRG2 cells was substantially lower than that of the control cells (Fig. 1B). Both $\left[{ }^{3} \mathrm{H}\right]$ thymidine incorporation assays and a BrdU incorporation assay indicated that the decreased growth rate was due to the impairment of DNA replication (Fig. $1 \mathrm{C}$ and $1 \mathrm{D})$. Wound-healing activity was also substantially decreased in the sh-DRG2 cells (Fig. 1E), confirming that DRG2 functions in the growth of HeLa cells. We then examined whether DRG2-depleted cells would be less tumorigenic in 

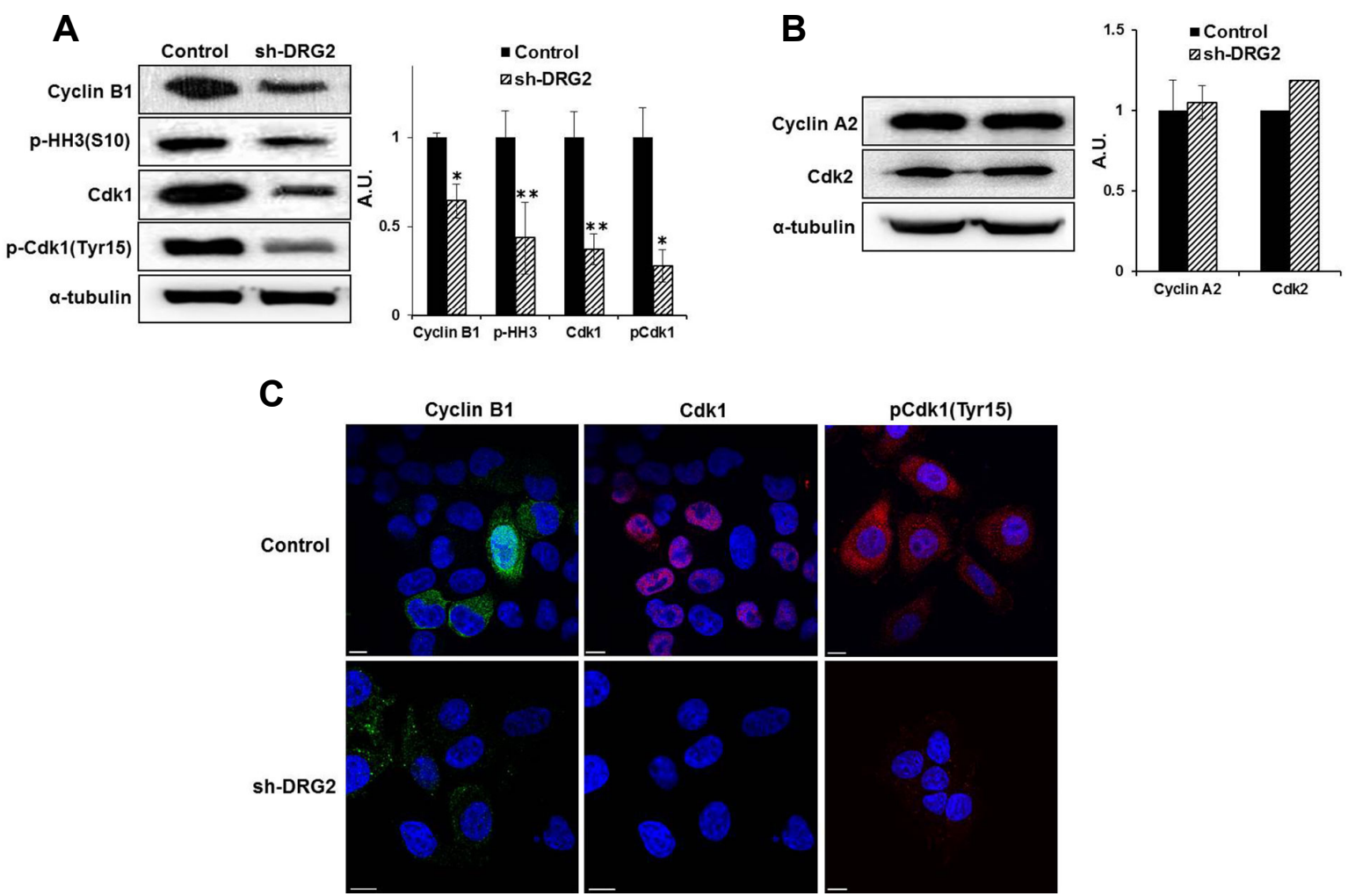

Fig. 3. Mitosis-promoting factors are decreased in sh-DRG2 cells. (A) The relative levels of cyclin B1, phospho Histone H3 (pHH3), and Cdk1 were significantly reduced in sh-DRG2 cells. Data are the means \pm S.E. of three independent experiments (Filled bar, control; slashed bar, sh-DRG2) $\left({ }^{*} p<0.05,{ }^{* *} p<0.01\right)$. (B) Cyclin B1 and Cdk1 were detected by immunofluorescence using a confocal microscope. Fluorescence images show markedly reduced expression of cyclin B1, Cdk1 and p-Cdk1(Tyr15) in sh-DRG2 cells compared with controls (Scale bar; $10 \mu \mathrm{m})$.

nude mice xenografts. Tumors appeared within 4 weeks in all of the animals of the control group, whereas no tumors developed in the mice injected with sh-DRG2 cells (Fig. 1F). These results together suggest that DRG2 functions in cell cycle progression.

DRG2 knockdown induced G2/ M arrest of the cell cycle To assess the consequences of DRG2 deficiency during the cell cycle, we investigated cells at different stages in the cell cycle. Flow cytometry analysis revealed that the population of G2/M phase cells increased in the sh-DRG2 cells (Fig. 2A). Furthermore, we noted that the level of DRG2 protein peaked at $12 \mathrm{~h}$ after synchronization, corresponding to the $\mathrm{M}$ phase (Fig. 2B), indicating that DRG2 is involved in mitosis.

\section{DRG2 regulates the expression of the mitosis regulating} factors

Entry into mitosis is triggered by progressive activation of cyclin B1/Cdk1 complexes at the end of G2 phase. To investigate whether the cycle defect in DRG2 depleted cell was associated with the G2/M checkpoint, we analyzed the expression of major cell cycle promoting factors, such as cyclin B1, Cdk1, phosphoCdk1 (p-Cdk1) and phospho-histone $\mathrm{H} 3(\mathrm{pHH} 3)$. Levels of cyclin $\mathrm{B} 1, \mathrm{Cdk} 1$ and $\mathrm{pHH} 3$ protein were reduced in the shDRG2 cells. Phosphorylated Cdk1 ( $p-C d k 1$ ), as well as, followed a similar pattern to dephosphorylated Cdk1, indicating that DRG2 is not involved in activating Cdk1 by phosphorylation (Fig. 3A). On the other hand, expression of G2 specific markers including cyclin A2 and Cdk2 was not affected by DRG2 depletion (Fig. 3B). Immunofluorescence analysis confirmed the reduced levels of cyclin $B 1, C d k 1$ and $p-C d k 1$ (Tyr15) protein in the sh-DRG2 cells (Fig. 3C). To exclude the possibility of offtarget effect of shRNA, we carried out the rescue experiment using shRNA resistant-hDRG2 clone. Decreased expression of cyclin B1, Cdk1 and pHH3 was able to restore after transfection of shRNA resistant-hDRG2 in sh-DRG2 cells (Supplementary Fig. $1 \mathrm{~A}$ ) and increased population of $\mathrm{G} 2 / \mathrm{M}$ phase by DRG2 depletion was also recovered by DRG2 rescue (Supplementary Fig. 1B). These data indicate that DRG2 deficiency decreases levels of proteins involved in the entry into mitosis.

Two kinases, Wee1 and Myt1, are responsible for the inhibitory phosphorylation of Cdk1 prior to mitotic entry. Cdk1 is also regulated by $\mathrm{p} 53$ via $\mathrm{p} 21$. p21 directly prevents mitosis by inactivating the mitotic cyclin-Cdk complex, and subsequent degradation of the mitotic cyclins further contributes to $\mathrm{G} 2$ arrest (Charrier-Savournin et al., 2004). To better understand the role of DRG2 in the G2/M transition, we measured p-Wee1, Myt1, and p21 protein levels in the DRG2-deficient cells and found that they were increased (Fig. 4A). Using immunofluorescence analysis to localize p21, we found that knockdown of DRG2 increased the proportion of p21-positive cells (Fig. 4B). Treatment with the Wee1-specific inhibitor, MK-1775, blocked cell 
A
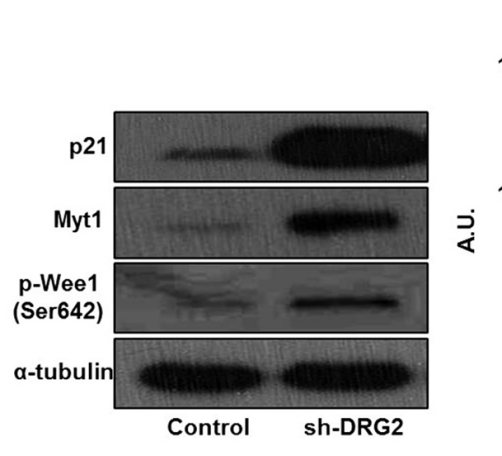

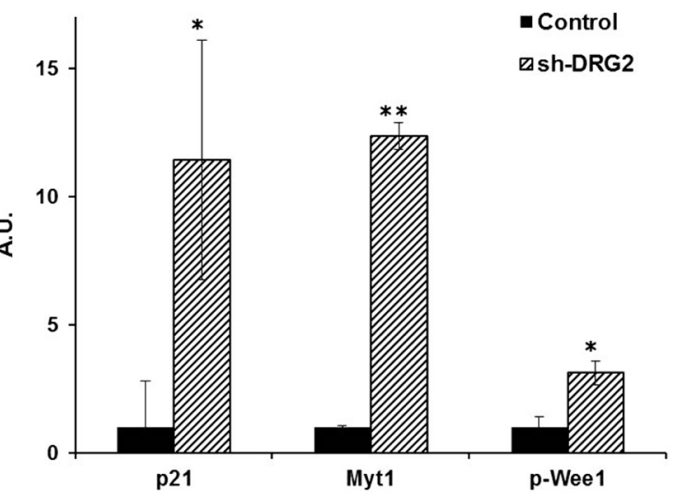

Fig. 4. Mitosis inhibitory factors are increased in sh-DRG2 cells. (A) The relative levels of p21, Myt1 and Wee1 increased significantly in shDRG2 cells. Data are means \pm S.E. of three independent experiments. (Filled bar; control, slashed bar; shDRG2) $\left({ }^{*} p<0.05,{ }^{* *} p<0.01\right)$. (B) Expression of p21 examined by immunofluorescence analysis. Expression of p21 increased in shDRG2 cells but its subcellular location was unchanged (Scale bar; 10 $\mu \mathrm{m})$.

B

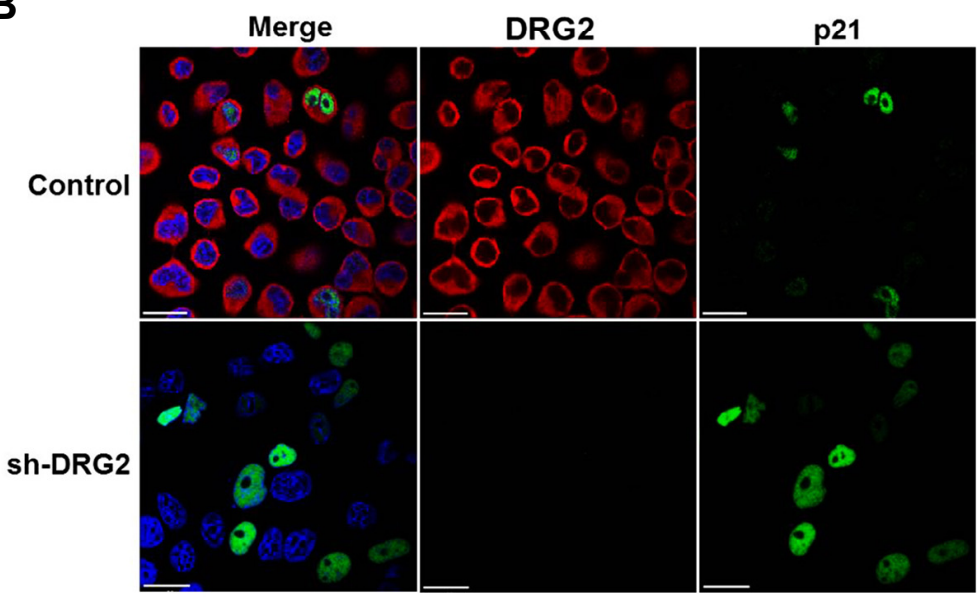

growth and phosphorylation of Cdk1 in control cells, but did not affect the sh-DRG2 cells (Supplementary Fig. 2), indicating that phosphorylation of Cdk1 is not associated with the cell cycle arrest due to absence of DRG2. These results together suggest that DRG2 deficiency upregulates the expression of p21, Wee1, and Myt1, which leads to downregulation of Cdk1 and cyclin B1 required for the entry to mitosis.

\section{DISCUSSION}

DRG2 was previously shown to be involved in cell cycle arrest at the G2/M checkpoint (Song et al., 2004), but its biochemical and physiological functions are largely unknown. In this study, DRG2 depleted cells exhibited increased G2/M phase arrest and were defective in cell proliferation. We investigated how DRG2 contributes to the G2/M transition. DRG2 deficiency resulted in down-regulation of the mitotic marker proteins Cdk1 and cyclin B1. The role of cyclin B1 is to induce the transition of cells from $G 2$ to $M$ phase, but it becomes dysregulated in cancer cells where overexpressed cyclin B1 can lead to uncontrolled cell growth by binding to its partner Cdks. When cyclin B1 is phosphorylated by Cdk1, it can be translocated to the nucleus, and progressive activation of cyclin B1/Cdk complexes coordinates entry into mitosis (Gavet and Pines, 2010). Different levels of cyclin B1/Cdk1 kinase activity trigger different mitotic events. A large number of proteins are phosphorylated by the cyclin B1/Cdk1 complex prior to mitotic entry. In addition, p-histone $\mathrm{H} 3$ phosphorylation at Ser-10 has traditionally been regarded as a marker for mitosis. The finding of markedly reduced histone $\mathrm{H} 3$ expression in the sh-DRG2 cells supports the view that the absence of DRG2 impairs mitotic progression. However, DRG2 is found only in the cytoplasm of HeLa cells. This suggests that Cdk1 expression induced by DRG2 is mediated by a certain transcription factor(s), which has not been revealed in this study.

We showed that DRG2 depletion increased levels of the phosphorylated Wee1, which is an important regulator of the G2/M checkpoint. In response to DNA damage, Wee1 becomes critical to restrain Cdk1 activity. In mammalian cells, translocation of Wee1 from the nucleus to the cytoplasm seems to be important for regulating cyclin B1/Cdk1 activity (Baldin and Ducommun, 1995; Oh et al., 2010). In S. pombe cells, the G2/M transition is inhibited by Wee1 (Masuda et al., 2011). To further analyze the mechanisms underlying the establishment of G2/M arrest in the sh-DRG2 cells, we inhibited Wee1 with the Wee1 inhibitor, MK1775, and found that this did not prevent phosphorylation of Cdk1 or alter the cell proliferation rate in the DRG2-deficient cells, whereas it reduced significantly phospho-Cdk1 levels and cell proliferation in control cells (Supplementary Fig. 2). Wee1 inhibition alone appears insufficient to trigger mitosis in the absence of Sphase perturbation, possibly because a critical level of Cdk1 activity is not achieved. Several positive feedback loops can 
amplify cyclin B/Cdk1 activation to ensure complete commitment to the mitotic state (Lindqvist et al., 2009). Myt1 and Wee1 kinases phosphorylate Cdk1 on T14 and Y15, thereby inhibiting cyclin B/Cdk1 activity. This suggests that DRG2 is not associated with the phosphorylation of Cdk1 by Wee 1 , although Wee1 may be involved in the $G 2$ arrest due to DRG2 deficiency. How DRG2 is regulated by Wee1 remains to be determined.

Our data show that genes encoding cell cycle-promoting proteins are down-regulated in DRG2-depleted HeLa cells but genes encoding cell cycle checkpoint genes are up-regulated. One of the checkpoint proteins, p21, is dramatically increased in the DRG2-depleted cells. p21 is required to maintain G2 arrest after DNA damage (Besson et al., 2008). Since p21 is important for fine-tuned control of Cdk1 activity in mitosis, its correct functioning facilitates smooth mitotic progression (Kreis et al., 2014). Expression of p21 positively correlates with the inhibition of proteins important for cell cycle progression, including Cdk1 and cyclin B1. In addition, nuclear p21 acts mainly as a tumor suppressor inducing cell cycle arrest, whereas cytoplasmic p21 behaves like an oncogene (Kreis et al., 2015). These observations suggest that the increased frequency of p21-positive nuclei observed in sh-DRG2 cells may be responsible for the augmentation of cell cycle delay.

Note: Supplementary information is available on the Molecules and Cells website (www.molcells.org).

\section{ACKNOWLEDGMENTS}

This research was supported by Basic Science Research Program (NRF-2014R1A1A3053240) through the National Research Foundation of Korea (NRF) funded by the Ministry of Science, ICT \& Future Planning and Priority Research Center Program (2009-0094050) and Basic Science Research Program (2013R1A1A4A01009559) through the National Research Foundation of Korea (NRF) funded by the Ministry of Education.

\section{REFERENCES}

Baldin, V., and Ducommun, B. (1995). Subcellular localisation of human wee1 kinase is regulated during the cell cycle. J. Cell Sci. 108( Pt 6), 2425-2432.

Besson, A., Dowdy, S.F., and Roberts, J.M. (2008). CDK inhibitors: cell cycle regulators and beyond. Dev. Cell 14, 159-169.

Cazzalini, O., Scovassi, A.I., Savio, M., Stivala, L.A., and Prosperi E. (2010). Multiple roles of the cell cycle inhibitor p21(CDKN1A) in the DNA damage response. Mutat. Res. 704, 12-20.

Charrier-Savournin, F.B., Chateau, M.T., Gire, V., Sedivy, J., Piette J., and Dulic, V. (2004). p21-Mediated nuclear retention of cyclin B1-Cdk1 in response to genotoxic stress. Mol. Biol Cell. 15 3965-3976.

Fisher, D., Krasinska, L., Coudreuse, D., and Novak, B. (2012). Phosphorylation network dynamics in the control of cell cycle transitions. J. Cell Sci. 125, 4703-4711.

Gavet, O., and Pines, J. (2010). Progressive activation of CyclinB1Cdk1 coordinates entry to mitosis. Dev. Cell 18, 533-543.

Ko, M.S., Lee, U.H., Kim, S.I., Kim, H.J., Park, J.J., Cha, S.J., Kim, S.B., Song, H., Chung, D.K., Han, I.S., et al. (2004). Overexpression of DRG2 suppresses the growth of Jurkat T cells but does not induce apoptosis. Arch. Biochem. Biophys. 422, 137-144.

Kreis, N.N., Sanhaji, M., Rieger, M.A., Louwen, F., and Yuan, J. (2014). p21Waf1/Cip1 deficiency causes multiple mitotic defects in tumor cells. Oncogene 33, 5716-5728.

Kreis, N.N., Louwen, F., and Yuan, J. (2015). Less understood issues: p21 in mitosis and its therapeutic potential. Oncogene 34, 1758-1767.

Lindqvist, A., Rodriguez-Bravo, V., and Medema, R.H. (2009). The decision to enter mitosis: feedback and redundancy in the mitotic entry network. J. Cell Biol. 185, 193-202.

Mani, M., Lee, U.H., Yoon, N.A., Kim, H.J., Ko, M.S., Seol, W., Joe, Y., Chung, H.T., Lee, B.J., Moon, C.H., et al. (2016). Developmentally regulated GTP-binding protein 2 coordinates Rab5 activity and transferrin recycling. Mol. Biol Cell. 27, 334-348.

Masuda, H., Fong, C.S., Ohtsuki, C., Haraguchi, T., and Hiraoka, Y. (2011). Spatiotemporal regulations of Wee1 at the G2/M transition. Mol. Biol Cell. 22, 555-569.

Oh, J.S., Han, S.J., and Conti, M. (2010). Wee1B, Myt1, and Cdc25 function in distinct compartments of the mouse oocyte to control meiotic resumption. J. Cell Biol. 188, 199-207.

Song, H., Kim, S.I., Ko, M.S., Kim, H.J., Heo, J.C., Lee, H.J., Lee, H.S., Han, I.S., Kwack, K., and Park, J.W. (2004). Overexpression of DRG2 increases G2/M phase cells and decreases sensitivity to nocodazole-induced apoptosis. J. Biochem. 135, 331-335. 